\title{
Moving Target Travelling Salesman Problem using Genetic Algorithm
}

\author{
Nitin S. Choubey \\ Professor \& Head, \\ Department of Computer Engineering \\ SVKM's NMIMS, Shirpur, Dhule, Maharashtra, India
}

\begin{abstract}
In this paper, the Moving Target Travelling Salesman Problem (MTTSP) is described. In MTTSP, several sites are required to be visited which are moving with constant velocity in different directions. The distance of the sites from origin, velocity and the angle of movement are known in advance. The goal is to find the fastest tour starting and ending at the origin which intercepts all the sites. The method implemented using genetic algorithm approach on the various data sets and the results are compared with greedy approach.
\end{abstract}

\section{General Terms}

Algorithm, complexity,

\section{Keywords}

Genetic Algorithm, Evolutionary Computation, Travelling Salesman Problem, Moving Target Travelling salesman problem, intercept, greedy method.

\section{INTRODUCTION}

The traveling salesman problem (TSP) was originated by the studies of two mathematicians Sir William Rowam Hamilton from Ireland and Thomas Penyngton Kirkman from Briton in the 18th century. The general form of the TSP is believed to be studied further Kalr Menger in Vienna and promoted by Hassler, Whitney \& Merrill at Princeton[2]. A detailed description about the connection between Menger \& Whitney, and the development of the TSP can be found in Schrijver, 2005[3].

Given a set of cities and the cost of travel (or distance) between each possible pairs, the objective of the TSP is to find the best possible way of visiting all the cities and returning to the starting point that minimize the travel cost (or travel distance)[1]. Given $n$ is the number of cities to be visited, the total number of possible routes covering all cities can be given as a set of feasible solutions of the TSP and is given as $(n-1) ! / 2$ [4]. In the classical TSP, the sites to be visited are stationary[10]. Motivated by practical application, Helvig et al.[4] introduced a time dependent generalization of TSP, referred as Moving Target TSP, where a pursuer must intercept in minimum time a set of targets which move with constant velocities (e.g., when a supply ship resupplies patrolling boats or when an aircraft must intercept a number of mobile ground units). Helvig et. al.[4] defined the MovingTarget travelling salesperson problem (MTTSP) as

"Given a set $S=\left\{s_{1}, \ldots s_{n}\right\}$ of targets, each $s_{i}$ moving at constant velocity $\mathrm{v}_{\mathrm{i}}$ from an initial position $\mathrm{p}_{\mathrm{i}}$, and given a pursuer starting at the origin and having maximum speed $\mathrm{v}>\left|\mathrm{v}_{\mathrm{i}}\right|$, find the fastest tour starting (and ending) at the origin, which intercepts all targets."

An instance of MTTSP with four targets to be visited is shown in the fig 1 . The start and end position of the pursuer is shown by flag. Helvig et. Al. has shown that the problem is NP-Hard. Several variants of MTTSP are given in [5][6].

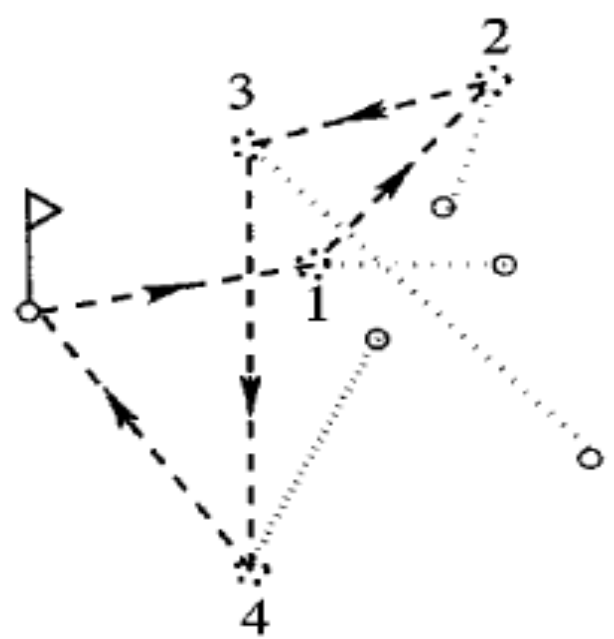

Fig 1 : MTTSP instance with four targets[4]

One of the current and best-known approaches for solving TSP problems is with the application of Evolutionary algorithms. These algorithms are based on naturally occurring phenomenon in nature, which are used to model computer algorithms. Potvin [11] has given the survey of Genetic Algorithm approaches for solving the general TSP. Most of evolutionary algorithm approaches try and find a solution to a particular problem, by recombining and mutating individuals in a society of possible solutions [6]. GA's are invented by John Holland in 1960s [7]. Holland's original goal was not to design algorithms to solve specific problems, but to formally study the phenomenon of adaptation as it occurs in nature and to develop ways in which the mechanisms of natural adaptation might be utilized into computer systems. Holland's 1975 book 'Adaptation in Natural and Artificial Systems' presented the GA as an abstraction of biological evolution and gave a theoretical framework for adaptation under the GA. Many problems in engineering and related areas require the simultaneous genetic optimization for a number of possibly competing objectives. These have been solved by combining the multiple objectives in to single scalar by the approach of linear combination. The combining coefficients, however, usually based on heuristic or guesswork can exert an unknown influence on the outcome of the optimization. A more satisfactory approach is to use the notion of Pareto optimality [8] in which an optimal set of solutions prescribe some surface 'The Pareto front' in the vector space of the objectives. For a solution on the Pareto front no objective can be improved without simultaneously degrading at least one other. 
Jindal et al.[9][16] attempted to solve an variant of MTTSP, with resupply when all the target are moving away from the origin, by using greedy methodology, where authors sorted the target in the order of $\mathrm{d}_{\mathrm{i}} / \mathrm{v}_{\mathrm{i}}$ to get the sequence of the sites to be visited, where $d_{i}$ is the distance of the $i^{\text {th }}$ target from origin and $\mathrm{v}_{\mathrm{i}}$ is the velocity of $\mathrm{i}^{\text {th }}$ target. The assumption made in this paper is that, although the target are either moving away from the original position of the pursuer or moving towards pursuer, they are also moving with some angle " $\theta$ " with respect to the origin[13][14][15]. It is assumed that the angle of movement also plays important role in determining the sequence to be visited for the optimal elapsed time. The MTTSP problem is addressed in this paper with the help of Genetic Algorithm. The solution generated with the help of Genetic algorithm is compared with that of generated with the help of greedy approach. Section 2 of the paper gives details of the suggested approach and the greedy method. Section 3 compares the result followed by concluding remarks in section 4.

\section{METHODOLOGY ADOPTED}

The suggested approach is divided in to two sub-section namely The generation of the sequence and calculation of Total Elapsed Time (TET) the simulation of the sequence to get total elapsed time for the sequence.

\subsection{Generation of sequence}

The suggested model uses genetic algorithm for generating the sequence. The answer generated with genetic algorithm is compared with that of greedy method. The methods are discussed in the following subsections.

\subsubsection{Genetic Algorithm methodology}

The Simple Genetic Algorithm (SGA) is adopted for generating the optimal sequence. A Simple Genetic Algorithm is given in fig. 2 .

1. Create initial random population.

2. Calculate fitness of the individuals in the population.

3. Repeat following steps until termination criteria is reached.

a. Select best fit from the current population and generate offspring.

b. Evaluate fitness of each offspring.

c. Replace weak individuals from the population with newly generated ones.

\section{Fig 2 : Simple Genetic Algorithm}

The GA produces successive generations of individuals by computing their "fitness" at each step and selecting the best of them, when the termination condition arises. Tournament selection method is used for selection of parent where two parents are chosen randomly and the best amongst them get a chance to be in the mating pool [12]. The termination criteria of user specified maximum number of generations is used. Two point order based crossover and swap single bit mutation is used for reproduction process. The sequence of the targets to be visited by the pursuer is used as chromosome. The working of the crossover and mutation operators is shown in Fig 3. Order based crossover is used to preserve the target in the chromosomes. The TET is used as fitness value for the chromosome sequence.

\subsubsection{Greedy Method}

The sequence of the sites to be visited is decided based on the ratio of $\mathrm{d}_{\mathrm{i}} / \mathrm{v}_{\mathrm{i}}$ and are scheduled in ascending and descending order of the ratio $d_{i} / v_{i}$.

\section{Two Point Order Based Crossover (TPOBC)}

$\begin{array}{cccccccccc}\text { P1 } & 1 & 2 & 3 & 4 & 5 & 6 & 7 & 8 & 9 \\ \text { P2 } & 9 & 8 & 7 & 6 & 5 & 4 & 3 & 2 & 1 \\ \text { Random Points } & 4 \text { \& } & 7 & & \uparrow & & & \uparrow & & \\ \text { Ch1 } & 1 & 2 & 3 & 4 & 7 & 6 & 5 & 8 & 9 \\ \text { Ch2 } & 9 & 8 & 7 & 6 & 3 & 4 & 5 & 2 & 1\end{array}$

\section{Swap Single Bit Mutation (SSBM)}

$\begin{array}{lccccccccc}\text { Child } & 1 & 2 & 3 & 4 & 5 & 6 & 7 & 8 & 9 \\ \text { Random Points (4,6) } & & \uparrow & & \uparrow & & & \\ \begin{array}{l}\text { Mutated } \\ \text { Child }\end{array} & 2 & 3 & 6 & 5 & 4 & 7 & 8 & 9\end{array}$

Fig. 3 : Reproduction operators used

\subsection{Calculation of Total Elapsed Time}

The TET is measure by adding the time required to visit each targets by the pursuer in the provided sequence. The process is shown in figure 4 .

1. Initialize the TET to zero, and target to visit as $\mathrm{s}_{\mathrm{i}}$ with
$\mathrm{i}=1$.
2. Calculate the time required to visit the target $\mathrm{s}_{\mathrm{i}}$ from
origin of the pursuer, say $\mathrm{t}_{\mathrm{i}}$. Update TET to TET $+\mathrm{t}_{\mathrm{i}}, \& \mathrm{i}$
to $\mathrm{i}+1$.
3. Calculate the time required to visit the target $\mathrm{s}_{\mathrm{i}}$ from the
place of the visit target $\mathrm{s}_{\mathrm{i}-1}$ by the pursuer, say $\mathrm{t}_{\mathrm{i}}$.
4. Update TET to TET $+\mathrm{t}_{\mathrm{i}}, \& \mathrm{i}$ to $\mathrm{i}+1$.
5. Repeat Steps $3 \& 4$ for all the targets in sequence.

Fig 4 : Calculation of TET

\section{Experimental Setup and Result Analysis}

The experiment is conducted with JDK 1.6 on an Intel Core $^{\mathrm{TM}} 2 \mathrm{CPU}$ with $2.66 \mathrm{GHZ}$ and $2 \mathrm{~GB}$ RAM. The Population size $=40$, Maximum number of generation $=500$, Crossover Rate $=0.8$ and mutation rate $=0.05$ is used for the purpose of simulation. Table 1, Table 2, Table 3 and Table 4 shows the four data sets used with four, six, eight and ten targets respectively for the experiment. The same data sets are used for Genetic Algorithm as well as for the greedy method. The results obtained after simulation are shown in Table 5, Table 6, Table 7 and Table 8 for the datasets with four, six, eight and ten targets respectively. The sample result for data set 10D3 is shown in fig 5. The initial position of the targets are shown by the red boxes. The path followed by the pursuer is shown by pink color whereas the track followed by the targets is shown by green color. The comparative chart for the total elapsed time taken by each method on various data sets is shown in fig 6. It is found that the time taken by the sequence generated by GA is much less than that of other methods. The result shows that the difference in the time taken for few targets is less as compared to that of more number of targets on various methods. The greedy methods does not take account of the of the target's direction of 
movement while deciding the sequence of the targets to be visited, where as in GA methods the final time taken by the pursuer is considered as a fitness function. It shows that there is need to incorporate the direction of targets movement apart from merely its direction and velocity only.

Table 1. Data Sets for Four targets

\begin{tabular}{|c|c|c|c|c|c|c|c|c|c|}
\hline Target Distance & \multicolumn{2}{|c|}{$\begin{array}{c}\text { Set 1, } \\
\mathbf{4 D}_{\mathbf{1}}\end{array}$} & \multicolumn{2}{|c|}{$\begin{array}{c}\text { Set 2, } \\
\mathbf{4 D}_{\mathbf{2}}\end{array}$} & \multicolumn{2}{|c|}{$\begin{array}{c}\text { Set 3, } \\
\mathbf{4 D}_{\mathbf{3}}\end{array}$} & \multicolumn{2}{|c|}{$\begin{array}{c}\text { Set 4, } \\
\mathbf{4 D}_{\mathbf{4}}\end{array}$} \\
\hline $\mathbf{S}_{\mathbf{i}}$ & $\mathbf{d}_{\mathbf{i}}$ & $\mathbf{v}_{\mathbf{i}}$ & $\boldsymbol{\theta}_{\mathbf{i}}$ & $\mathbf{v}_{\mathbf{i}}$ & $\boldsymbol{\theta}_{\mathbf{i}}$ & $\mathbf{v}_{\mathbf{i}}$ & $\boldsymbol{\theta}_{\mathbf{i}}$ & $\mathbf{v}_{\mathbf{i}}$ & $\boldsymbol{\theta}_{\mathbf{i}}$ \\
\hline 1 & 303 & 27 & 80 & 17 & 80 & 17 & 80 & 9 & 80 \\
\hline 2 & 515 & 8 & 150 & 23 & 150 & 23 & 150 & 3 & 150 \\
\hline 3 & 596 & 20 & 30 & 10 & 30 & 10 & 30 & 16 & 30 \\
\hline 4 & 497 & 28 & 45 & 17 & 45 & 17 & 45 & 1 & 45 \\
\hline
\end{tabular}

Table 2. Data Sets for Six targets

\begin{tabular}{|l|c|c|c|c|c|c|c|c|c|}
\hline Target Distance & \multicolumn{2}{|c|}{$\begin{array}{c}\text { Set 1, } \\
\mathbf{6 D}_{\mathbf{1}}\end{array}$} & \multicolumn{2}{|c|}{$\begin{array}{c}\text { Set 2, } \\
\mathbf{6 D}_{\mathbf{2}}\end{array}$} & \multicolumn{2}{c|}{$\begin{array}{c}\text { Set 3, } \\
\mathbf{6 D}_{\mathbf{3}}\end{array}$} & \multicolumn{2}{|c|}{$\begin{array}{c}\text { Set 4, } \\
\mathbf{6 D}_{\mathbf{4}}\end{array}$} \\
\hline $\mathbf{S}_{\mathbf{i}}$ & $\mathbf{d}_{\mathbf{i}}$ & $\mathbf{v}_{\mathbf{i}}$ & $\boldsymbol{\theta}_{\mathbf{i}}$ & $\mathbf{v}_{\mathbf{i}}$ & $\boldsymbol{\theta}_{\mathbf{i}}$ & $\mathbf{v}_{\mathbf{i}}$ & $\boldsymbol{\theta}_{\mathbf{i}}$ & $\mathbf{v}_{\mathbf{i}}$ & $\boldsymbol{\theta}_{\mathbf{i}}$ \\
\hline 1 & 303 & 2 & 80 & 6 & 80 & 26 & 80 & 27 & 80 \\
\hline 2 & 515 & 25 & 150 & 22 & 150 & 12 & 150 & 7 & 150 \\
\hline 3 & 596 & 14 & 30 & 25 & 30 & 23 & 30 & 19 & 30 \\
\hline 5 & 497 & 29 & 45 & 6 & 45 & 6 & 45 & 20 & 45 \\
\hline 6 & 527 & 22 & 60 & 4 & 60 & 5 & 60 & 27 & 60 \\
\hline
\end{tabular}

Table 3. Data Sets for Eight targets

\begin{tabular}{|l|l|c|c|c|c|c|c|c|c|}
\hline Target & Distance & $\begin{array}{c}\text { Set 1, } \\
\mathbf{8 D}_{\mathbf{1}}\end{array}$ & \multicolumn{2}{|c|}{$\begin{array}{c}\text { Set 2, } \\
\mathbf{8 D}_{\mathbf{2}}\end{array}$} & \multicolumn{2}{|c|}{$\begin{array}{c}\text { Set 3, } \\
\mathbf{8 D}_{\mathbf{3}}\end{array}$} & \multicolumn{2}{|c|}{$\begin{array}{c}\text { Set 4, } \\
\mathbf{8 D}_{\mathbf{4}}\end{array}$} \\
\hline $\mathbf{S}_{\mathbf{i}}$ & $\mathbf{d}_{\mathbf{i}}$ & $\mathbf{v}_{\mathbf{i}}$ & $\boldsymbol{\theta}_{\mathbf{i}}$ & $\mathbf{v}_{\mathbf{i}}$ & $\boldsymbol{\theta}_{\mathbf{i}}$ & $\mathbf{v}_{\mathbf{i}}$ & $\boldsymbol{\theta}_{\mathbf{i}}$ & $\mathbf{v}_{\mathbf{i}}$ & $\boldsymbol{\theta}_{\mathbf{i}}$ \\
\hline 1 & 303 & 16 & 80 & 4 & 00 & 8 & 80 & 14 & 80 \\
\hline 2 & 515 & 26 & 150 & 3 & 150 & 17 & 150 & 28 & 150 \\
\hline 3 & 596 & 27 & 30 & 4 & 30 & 9 & 30 & 17 & 30 \\
\hline 5 & 497 & 1 & 45 & 11 & 45 & 7 & 45 & 22 & 45 \\
\hline 7 & 419 & 26 & 136 & 30 & 168 & 4 & 32 & 23 & 14 \\
\hline 8 & 281 & 10 & 104 & 13 & 334 & 7 & 42 & 17 & 28 \\
\hline
\end{tabular}

Table 4. Data Sets for Ten targets

\begin{tabular}{|l|l|c|c|c|c|c|c|c|c|}
\hline Target & Distance & \multicolumn{2}{|c|}{$\begin{array}{c}\text { Set 1, } \\
\mathbf{1 0 D}_{\mathbf{1}}\end{array}$} & \multicolumn{2}{|c|}{$\begin{array}{c}\text { Set 2, } \\
\mathbf{1 0 D}_{\mathbf{2}}\end{array}$} & \multicolumn{2}{c|}{$\begin{array}{c}\text { Set 3, } \\
\mathbf{1 0 D}_{\mathbf{3}}\end{array}$} & \multicolumn{2}{|c|}{$\begin{array}{c}\text { Set 4, } \\
\mathbf{1 0 D}_{\mathbf{4}}\end{array}$} \\
\hline $\mathbf{S}_{\mathbf{i}}$ & $\mathbf{d}_{\mathbf{i}}$ & $\mathbf{v}_{\mathbf{i}}$ & $\boldsymbol{\theta}_{\mathbf{i}}$ & $\mathbf{v}_{\mathbf{i}}$ & $\boldsymbol{\theta}_{\mathbf{i}}$ & $\mathbf{v}_{\mathbf{i}}$ & $\boldsymbol{\theta}_{\mathbf{i}}$ & $\mathbf{v}_{\mathbf{i}}$ & $\boldsymbol{\theta}_{\mathbf{i}}$ \\
\hline 1 & 303 & 15 & 80 & 3 & 80 & 1 & 80 & 20 & 80 \\
\hline 3 & 515 & 3 & 150 & 26 & 150 & 5 & 150 & 19 & 150 \\
\hline 4 & 497 & 11 & 45 & 16 & 45 & 3 & 45 & 18 & 45 \\
\hline 5 & 527 & 13 & 60 & 10 & 60 & 26 & 60 & 5 & 60 \\
\hline 7 & 288 & 30 & 283 & 26 & 356 & 39 & 15 & 23 & 313 \\
\hline 8 & 311 & 3 & 37 & 20 & 309 & 29 & 284 & 22 & 15 \\
\hline 9 & 203 & 36 & 179 & 28 & 281 & 25 & 171 & 33 & 11 \\
\hline 10 & 462 & 16 & 86 & 4 & 15 & 15 & 145 & 30 & 95 \\
\hline
\end{tabular}

Table 5. Results for set with four targets

\begin{tabular}{|c|c|c|c|c|c|c|}
\hline \multirow{3}{*}{$\begin{array}{l}\text { Data } \\
\text { Sets }\end{array}$} & \multirow{2}{*}{\multicolumn{2}{|c|}{ GA Method }} & \multicolumn{4}{|c|}{ Greedy Method } \\
\hline & & & \multicolumn{2}{|c|}{ Di/vi, Ascending } & \multicolumn{2}{|c|}{ Di/vi, Descending } \\
\hline & Solution & TET & Solution & TET & Solution & TET \\
\hline $4 D_{1}$ & $1-2-3-4$ & 24911 & $2-3-4-1$ & 25821 & $1-4-3-2$ & 26534 \\
\hline $4 D_{2}$ & $1-4-2-3$ & 24453 & $3-4-2-1$ & 24860 & $1-2-4-3$ & 24621 \\
\hline $4 D_{3}$ & $1-4-2-3$ & 26056 & $4-2-3-1$ & 28349 & $1-3-2-4$ & 27651 \\
\hline $4 D_{4}$ & $1-2-3-4$ & 24713 & $1-3-2-4$ & 25824 & $4-2-3-1$ & 26293 \\
\hline
\end{tabular}

Table 6. Results for set with Six targets

\begin{tabular}{|c|c|c|c|c|c|c|}
\hline \multirow{2}{*}{$\begin{array}{c}\text { Data } \\
\text { Sets }\end{array}$} & \multicolumn{2}{|c|}{ GA Method } & \multicolumn{4}{c|}{ Greedy Method } \\
\cline { 2 - 7 } & \multicolumn{2}{|c|}{ Solution } & TET & Solution & TET & \multicolumn{2}{c|}{ Solution } & TET \\
\hline $\mathbf{6 D}_{\mathbf{1}}$ & $\begin{array}{c}6-1-2-4- \\
3-5\end{array}$ & 26458 & $\begin{array}{c}6-4-2-5- \\
3-1\end{array}$ & 30035 & $\begin{array}{c}1-3-5-2- \\
4-6\end{array}$ & 33685 \\
\hline $\mathbf{6 D}_{\mathbf{2}}$ & $\begin{array}{c}6-5-3-4- \\
2-1\end{array}$ & 27945 & $\begin{array}{c}2-3-1-4- \\
6-5\end{array}$ & 43354 & $\begin{array}{c}5-6-4-1- \\
3-2\end{array}$ & 49389 \\
\hline $\mathbf{6 D}_{\mathbf{3}}$ & $\begin{array}{c}6-5-3-2- \\
4-1\end{array}$ & 28024 & $\begin{array}{c}1-6-3-2- \\
4-5\end{array}$ & 36692 & $\begin{array}{c}5-4-2-3- \\
6-1\end{array}$ & 49461 \\
\hline $\mathbf{6 D}_{\mathbf{4}}$ & $\begin{array}{c}6-1-2-5- \\
3-4\end{array}$ & 27919 & $\begin{array}{c}1-5-4-6- \\
3-2\end{array}$ & 48437 & $\begin{array}{c}2-3-6-4- \\
5-1\end{array}$ & 48527 \\
\hline
\end{tabular}

Table 7. Results for set with eight targets

\begin{tabular}{|c|c|c|c|c|c|c|}
\hline \multirow{3}{*}{$\begin{array}{l}\text { Data } \\
\text { Sets }\end{array}$} & \multirow{2}{*}{\multicolumn{2}{|c|}{ GA Method }} & \multicolumn{4}{|c|}{ Greedy Method } \\
\hline & & & \multicolumn{2}{|c|}{ Di/vi, Ascending } & \multicolumn{2}{|c|}{ Di/vi, Descending } \\
\hline & Solution & TET & Solution & TET & Solution & TET \\
\hline $8 D_{1}$ & \begin{tabular}{|}
$6-5-3-2-$ \\
$4-7-1-8$
\end{tabular} & 29789 & $\begin{array}{c}6-7-1-2- \\
3-5-8-4\end{array}$ & 42326 & $\begin{array}{l}4-8-5-3- \\
2-1-7-6\end{array}$ & 61764 \\
\hline $8 D_{2}$ & $\begin{array}{c}8-7-4-3- \\
5-2-6-1\end{array}$ & 28526 & $\begin{array}{l}7-5-6-8- \\
4-1-3-2\end{array}$ & 47136 & $\begin{array}{c}2-3-1-4- \\
8-6-5-7\end{array}$ & 60615 \\
\hline $8 D_{3}$ & \begin{tabular}{|c|}
$8-6-5-3-$ \\
$2-4-7-1$ \\
\end{tabular} & 26880 & $\begin{array}{l}6-2-8-1- \\
3-4-7-5 \\
\end{array}$ & 39875 & $\begin{array}{c}5-7-4-3- \\
1-8-2-6\end{array}$ & 46494 \\
\hline $8 D_{4}$ & $\begin{array}{c}8-6-5-3- \\
4-2-7-1\end{array}$ & 29726 & $\begin{array}{c}7-2-6-1- \\
4-8-5-3\end{array}$ & 52419 & $\begin{array}{c}3-5-8-4- \\
1-6-2-7\end{array}$ & 64171 \\
\hline
\end{tabular}


Table 8. Results for set with ten targets

\begin{tabular}{|c|c|c|c|c|c|c|}
\hline \multirow{3}{*}{$\begin{array}{l}\text { Data } \\
\text { Sets }\end{array}$} & \multirow{2}{*}{\multicolumn{2}{|c|}{ GA Method }} & \multicolumn{4}{|c|}{ Greedy Method } \\
\hline & & & \multicolumn{2}{|c|}{ Di/vi, Ascending } & \multicolumn{2}{|c|}{\begin{tabular}{|l} 
Di/vi, Descending \\
\end{tabular}} \\
\hline & Solution & TET & Solution & TET & Solution & TET \\
\hline $10 D_{1}$ & \begin{tabular}{|c|}
$9-7-6-10-$ \\
$2-5-3-4-$ \\
$1-8$
\end{tabular} & 29862 & \begin{tabular}{|c|}
$9-7-6-1-$ \\
$10-5-4-3-$ \\
$8-2$
\end{tabular} & 45654 & \begin{tabular}{|c|}
$2-8-3-4-$ \\
$5-10-1-6-$ \\
$7-9$ \\
\end{tabular} & 67522 \\
\hline $10 D_{2}$ & \begin{tabular}{|c|}
$9-8-7-6-$ \\
$10-5-3-4-$ \\
$2-1$
\end{tabular} & 30878 & $\begin{array}{c}9-7-8-2- \\
3-6-4-5- \\
10-1\end{array}$ & 42623 & \begin{tabular}{|c|}
$1-10-5-4-$ \\
$6-3-2-8-$ \\
$7-9$ \\
\end{tabular} & 77921 \\
\hline $10 D_{3}$ & \begin{tabular}{|c|}
$8-7-6-5-$ \\
$3-2-4-10-$ \\
$1-9$
\end{tabular} & 30076 & $\begin{array}{c}7-9-8-10- \\
5-3-2-4- \\
1-6\end{array}$ & 44276 & \begin{tabular}{|c|}
$6-1-4-2-$ \\
$3-5-10-9-$ \\
$8-7$ \\
\end{tabular} & 66117 \\
\hline $10 D_{4}$ & \begin{tabular}{|c|}
$9-7-1-6-$ \\
$2-4-10-3-3-$ \\
$5-8$
\end{tabular} & 30929 & \begin{tabular}{|c|}
$9-8-1-10-$ \\
$7-3-2-4-$ \\
$6-5$
\end{tabular} & 54279 & \begin{tabular}{|c|}
$5-6-4-2-$ \\
$3-7-10-1-$ \\
$8-9$
\end{tabular} & 66543 \\
\hline
\end{tabular}

\section{CONCLUSION}

In this paper, Moving Target TSP is studied. The method is implemented with Genetic Algorithm methods. The result of the experiment tested on the various datasets. The results generated by Genetic Algorithm method found to be more effective than generated by greedy method. There is need to find the effective solution by using analytical method for incorporating the target's direction of movement.

\section{ACKNOWLEDGMENTS}

Author thanks Mrs. Susheela Choubey and Prof. P. S. Gawande for their valuable time and contribution given while the drafting this paper. Author also thank to the reviewer for their expertise and keen review.

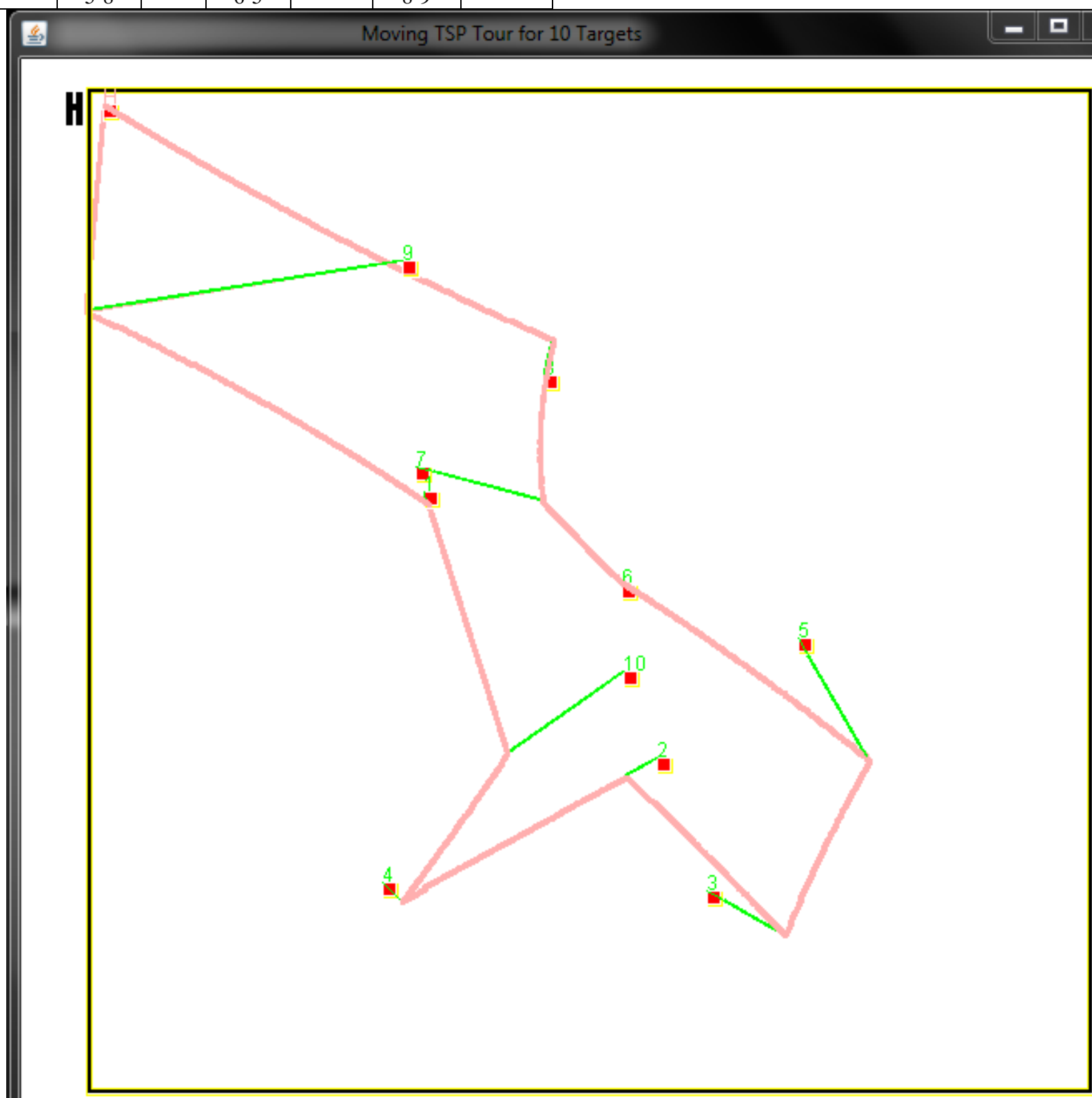

Fig 5 : Simulation Result for data set 10D3 


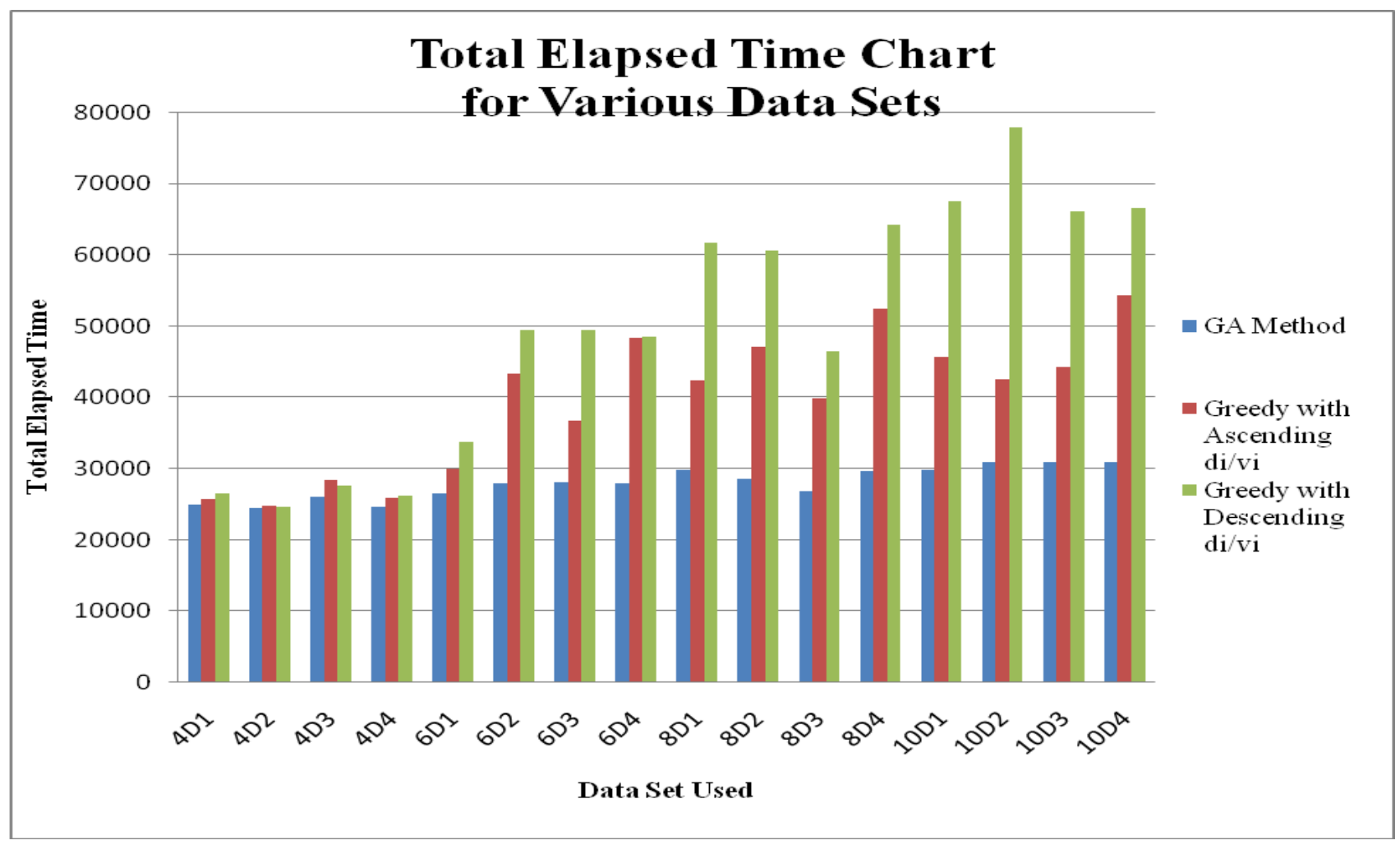

Fig 6 : Comparison chart

\section{REFERENCES}

[1] Horowitz E., Sahani S, and Rajasekaran S, 2007. Fundamentals of Computer Algorithm, University Press.

[2] Rajesh Matai, Surya Singh and Murari Lal Mittal (2010). Traveling Salesman Problem: an Overview of Applications, Formulations, and Solution Approaches, Traveling Salesman Problem, Theory and Applications, Prof. Donald Davendra (Ed.), ISBN: 978-953-307-426-9, InTech, Available from: http://www.intechopen.com/books/traveling-salesmanproblem-theory-and-applications/travelingsalesmanproblem an-overview-of-applicationsformulations-and-solution-approaches.

[3] Schrijver, A., 2005. On the History of Combinatorial Optimization (Till 1960). In K. Aardal, G. L. Nemhauser, \& R. Weismantel (Eds.), Handbooks in operations research and management science, 12, Amsterdam: Elsevier.

[4] Helvig C. S., Gabriel Robins, and Alex Zelikovsky, 2003. The Moving-target travelling salseman problem, Journal of Algorithms 49 (2003), 153-174.

[5] Helvig C. S. Gabriel Robins, and Alex Zelikovsky, C. H. Helvig, G. Robins, and A. Zelikovsky. "Moving Target TSP and Related Problems", G. Bilardi et Al. (Eds.): ESA'98, LNCS 1461, pp 435-1998.

[6] D. P. Guy, Evolutionary Computing as a Tool for Grammar Development, CNTS - Language Technology Group, UIA - University of Antwerp, Antwerp Belgium, GECCO 2003 LNCS 2723, Springer-Verlag, Berlin, Heidelberg, 2003, pp. 549-560.

[7] J. H. Holland, Adoption in Natural and Artificial System, University of Michigan Press, Ann Arbor, MI, 1975.
[8] D. E. Goldberg, Genetic Algorithms in Search, Optimizations and Machine Learn-ing, Addison-Wesley, 1989.

[9] Jindal Pawan, Amit Kumar, and Shishir Kumar, 2011, "Dynamic Version of Travelling Salseperson Problem", International Journal of Computer Applications (0975 8887), Volume 19- No.1, April 2011.

[10] Choubey Nitin S., 2012, "Solving TSP using DARO", International Journal of Computer Applications (09758887), Volume 58-No.9, November 2012.

[11] Potvin J. Y., 1996, "Geneti Algorithms for the Travelling Salseman Problem", Annals of Operation Research, 339370, 1996.

[12] Sivanandam, Deepa, 2008, "Introduction to Genetic Algorithm”, Springer, 2008.

[13] Bartley Cristopher, 2004, "Closed Form Guidance Laws for Intercepting Moving Targets" Master's thesis, Rice University, Houstons, Texas, April 2004.

[14] Gabrial Jacob Diaz, Flip Phillips and Brett R. Fajen, 2009, "Intercepting Moving Targets: a Little Foresight Helps a Lot" Springer-Verlag 2009.

[15] Jason R. Looker, 2008, "Minimum Paths to Interception of a Moving Targets when Constrained by Turning Radius", DSTO Defence Science and Technology Organisation, Australia, December 2008.

[16] Jindal Pawan, Amit Kumar, and Shishir Kumar, 2011, "Multiple Target Intercepting Travelling Salseperson Problem", International Journal of Computer Science and Technology, (0976 - 8491), Volume 2- Issue 2, June 2011. 